\title{
Interplay of Socio-Cultural and Environmental Factors on Microbial Contamination of Food in Samaru, Kaduna State, Nigeria
}

\author{
Beatty V. Maikai* and Mujtaba A. Salman
}

Ahmadu Bello University, Zaria, Nigeria

\section{Objective}

To study the effect of socio-cultural and environmental factors on microbial contamination of food and the understanding of foodrelated risks, food safety knowledge and practices

\section{Introduction}

In most disadvantaged communities in Northern Nigeria, adolescent girls engage in economic activities so that they can save money for household items to be bought for them when they are given out for marriage. These girls right from before they reach teenage age hawk items which include ready-to-eat foods $\left({ }^{1}\right)$. Various socio-cultural and environmental factors reinforce vulnerability of foods to microorganisms. Food safety awareness, knowledge and practices among food vendors can be affected by interplay between individual and outdoor factors. Teenagers engage in hawking food without understanding food-related risks for the preservation of their health and the health of others. Food hygiene is the conditions and measures necessary to ensure the safety of food from production to consumption. Lack of adequate food hygiene can lead to foodborne diseases and death of the consumer $\left({ }^{2}\right)$. Mishandling of food can occur during food preparation, handling and storage; and studies show that consumers have inadequate knowledge about measures needed to prevent food-borne illness $\left({ }^{3}\right)$. There are a number of factors which are likely to contribute to outbreaks of food-borne illness in the home, including a raw food supply that may be contaminated, a lack of food safety knowledge among the general public, mistakes in food handling and preparation at home $\left({ }^{4}\right)$.

\section{Methods}

Fifty-four food samples comprising Rice and Beans meals as well as local delicacies made from maize, soyabeans and others were collected within Samaru in Kaduna State, Nigeria. The samples were pre-enriched and enriched with buffered peptone water and tetrationate broth respectively before plating on Desoxycholate Citrate Agar. Structured questionnaires were also administered to the food vendors in form of an interview. Questions covered method of food preparation, reheating prior to sale, food safety measures, awareness of food safety, knowledge and behavioral practices that may enhance foodborne illness.

\section{Results}

Of the 54 samples, $20(37 \%)$ were non-lactose fermenters. Two $(10 \%)$ out of these 20 suspected organisms showed reactions consistent with that of Salmonella species upon characterizing them biochemically. The remaining $18(90 \%)$ that were not Salmonella showed reactions typical of Proteus 5 (9.3\%), Citrobacter 8 (14.8\%), E. coli and Arizona spp 1 (1.9\%) each and 3 (5.6\%) unidentified spp. For food-related risks, a low level of awareness and bad behavioural practices such as playing around food sale points, exposure of food to dusty and unhygienic environment, not washing of utensils and cutleries with clean water prior to serving consumers, hand contact with served foods and a low level of perceived vulnerability to foodborne illness were observed. Particular lack of knowledge was identified regarding the impact of temperature on microorganisms as the food sold were either already cold or lukewarm and ignorance on possible health hazards with unprotected wounds on their hands as was seen on some. Though more females $43(79.6 \%)$ were seen selling foods than males $11(37.0 \%)$, but the foods sold by male vendors were more contaminated probably because factors observed in females were more elaborate in males.

\section{Conclusions}

Understanding of food safety practices is helpful in reducing food-borne illness. Preventive health strategies that make use of good behavioral and hygienic environment should be targeted. Food vendors should be given the time, tools and training necessary to facilitate proper food handling practices to know basic food safety measures early in life.

\section{Keywords}

Food; Safety; Microorganisms; Environment; culture

\section{Acknowledgments}

Laboratory staff and the Department of Veterinary Public Health and Preventive Medicine, Ahmadu Bello University, Zaria, Nigeria for laboratory materials and technical assistance

\section{References}

1.Mercy corps. http://www.mercycorps.org/ sites/default/files/Mercy\%20 Corps\%20February \%20 2013\%20-\%20 Adolescent\%20Girls\%20 in\%20Northern\%20Nigeria. accessed September 2015)

2. Andrej O, Mojca J, Peter R. Food safety awareness, knowledge and practices among students in Slovenia. Food Cont 2014; 42: 144-151

3. WHO Report. Reducing risks, promoting healthy life. Geneva, World Health Organization (http://www.who.int/whr/2002/, accessed March 2005).

4. Kaferstein F. Actions to reverse the upward curve of foodborne illness. Food Cont 2003; 14 (2): 101-109

\section{${ }^{*}$ Beatty V. Maikai}

E-mail: Beatt18@yahoo.com 